\title{
Embalses de retención y canales verdes: herramientas para el control de inundaciones y la planificación urbana. Caso de estudio: municipio de Turbaco, Bolívar
}

Retention reservoirs and green canals: tools for flood control and urban planning Case study: Turbaco municipality, Bolivar

Milton Guerrero Pájaro, ${ }^{1}$ M.Sc.

${ }^{1}$ Universidad del Norte, Barranquilla, Colombia. Correspondencia: mguerrero01@gmail.com

Recibido: 13 abril 2016. Aceptado: 19 de mayo de 2016.

Guerrero, M. (2016) Embalses de retención y canales verdes: herramientas para el control de inundaciones y la planificación urbana. Caso de estudio: municipio de Turbaco, Bolívar. Procesos urbanos Número 3, Ene-Dic. 108-119. Doi: $10.21892 / 2422085 X .271$

\section{RESUMEN}

La rapidez en el crecimiento de nuestras ciudades y municipios, y el aumento vertiginoso en la demanda de suelo urbanizable, ha originado la impermeabilización del suelo urbanizado, lo que ha producido un aumento en los caudales de escorrentía. Por otro lado, el cambio climático impone nuevos desafíos en materia de gestión del drenaje pluvial para nuestras ciudades y municipios. El municipio de Turbaco vive una problemática en materia de drenaje pluvial, por causa del aumento en la demanda de suelo urbanizable y por la impermeabilización del suelo urbanizado. Los embalses de retención y las canales verdes surgen como una alternativa para la gestión de las aguas pluviales. Estos sistemas son de fácil adaptación al medio y son soluciones que van en favor del medio ambiente, al tiempo que constituyen parte del paisaje urbano.

Palabras clave: Inundaciones, embalses, canales verdes, análisis hidrológico, planificación urbana.

\section{ABSTRACT}

The rapid growth of our cities and municipalities, and the rapid increase in the demand for urbanizable land, has led to the waterproofing of urbanized land, which has led to an increase in runoff flows. On the other hand, climate change imposes new challenges in the management of storm drainage for our cities and municipalities. The municipality of Turbaco lives a problem in the matter of rainwater drainage, due to the increase in the demand of urbanizable land and the waterproofing of the urbanized land. Retention reservoirs and green channels emerge as an alternative to stormwater management. These systems are easy to adapt to the environment and are solutions that are in favor of the environment, while being part of the urban landscape.

Keywords: Floods, reservoirs, green channels, hydrological analysis, urban planning. 
Guerrero, M. - Embalses de retención y canales verdes: herramienta para el control de innundaciones.

\section{INTRODUCCIÓN}

El municipio de Turbaco se encuentra localizado al norte del departamento de Bolívar, a unos $10 \mathrm{kms}$. aproximadamente de la ciudad de Cartagena de Indias. En los últimos 10 años el casco urbano de este municipio se ha visto presionado por la demanda de suelo urbano para el desarrollo de conjuntos residenciales. Con los procesos de urbanización, el suelo natural ha sido impermeabilizado y como consecuencia de lo anterior han aumentado los caudales producto de la escorrentía pluvial. A esta situación se suma la falta de planificación de algunos sectores, en especial del sector nororiental del casco urbano del municipio, el cual ha enfrentado inundaciones sistemáticas. Como consecuencia de las inundaciones se han presentado problemas de salud, económicos, de movilidad, ambientales entre otros. Este municipio, por su posición geográfica, altura con respecto al nivel del mar y su vegetación es muy deseado por los urbanizadores. Este interés ha crecido al punto que diferentes proyectos urbanísticos han sido ejecutados en el sector. Los problemas de inundación han propiciado la búsqueda de soluciones que ambientalmente se incorporen al paisaje urbano natural. Los embalses de retención y las cunetas verdes hacen parte de los sistemas de drenaje sostenible (SUDS) que además de cumplir con la función de controlar los caudales picos producto de la escorrentía pluvial, se incorporan fácilmente al paisaje natural del municipio. Lo que se propone es que dentro de los proyectos urbanísticos que se proyectan, se incluyan los embalses de retención y las cunetas verdes con elementos de planificación urbana del municipio, logrando de esta forma reproducir el sistema hidrológico natural y minimizar el impacto de las inundaciones.

Este no es un problema en particular del casco urbano del municipio de Turbaco, diversas poblaciones de Colombia presentan problemas de inundación, producto de la impermeabilización del suelo como consecuencia de las urbanizaciones. Esta situación se incrementa con los nuevos retos que impone el cambio climático en el manejo y gestión del drenaje pluvial.
En el mundo los sistemas de drenaje sostenible son muy conocidos y en especial los embalses de retención; experiencias como la de la ciudad de Madrid donde se construyó un gran estanque de tormenta con el objetivo de desarrollar el plan de mejora de la calidad de agua del rio Manzanares habla del buen funcionamiento del sistema (Ayuntamiento de Madrid - Convive Madrid Río).

\section{CARACTERÍSTICA DEL ÁREA DE ESTUDIO}

El municipio de Turbaco actualmente no cuenta con un sistema de alcantarillado pluvial que le permita gestionar las aguas provenientes de la escorrentía superficial. Dentro de la cabecera municipal existen dos microcuencas que recogen la escorrentía pluvial, una de ellas drena hacia la ciénaga de la Virgen, localizada en la ciudad de Cartagena y la otra drena hacia el canal del Dique por medio del arroyo Grande. Dentro de los arroyos que hacen parte del municipio están: el arroyo Grande, Cucumán, Remanganagua, Mameyal que drenan hacia el canal del Dique y el arroyo Matute que lo hace hacia la ciénaga de la Virgen. El clima promedio al año del municipio es de $30^{\circ} \mathrm{C}$ y la altura promedio con respecto al nivel medio del mar es de $200 \mathrm{~m}$. El suelo se caracteriza por presencia de calizas porosas y solubles, lo que permite la infiltración y la conducción de agua hacia otros estratos del suelo. La cabecera municipal cuenta con 48 barrios organizados en 6 comunas (Alcaldía municipal de Turbaco, 2002). Los barrios más afectados por los eventos de inundación durante el último periodo invernal de los años 2010 y 2011 fueron La Victoria, Villa Leydi, El Ají, Fátima, Los Inválidos y Plan Parejo (Cardique \& Universidad de Cartagena 2011).

\section{PROBLEMAS DE INUNDACIÓN}

El casco urbano del municipio tiene un área de $7,25 \mathrm{~km}^{2}$ de los cuales el $20 \%$ se encuentra en riesgo de inundación (Figura 1). El origen de las inundaciones involucra varios factores, uno de los más importantes es la falta de un sistema de drenaje pluvial, otro es la impermeabilización del suelo producto

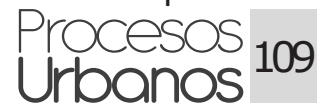




\section{ÁREAS DE INUNDACIÓN - CASCO URBANO DEL MUNICIPIO DE TURBACO}

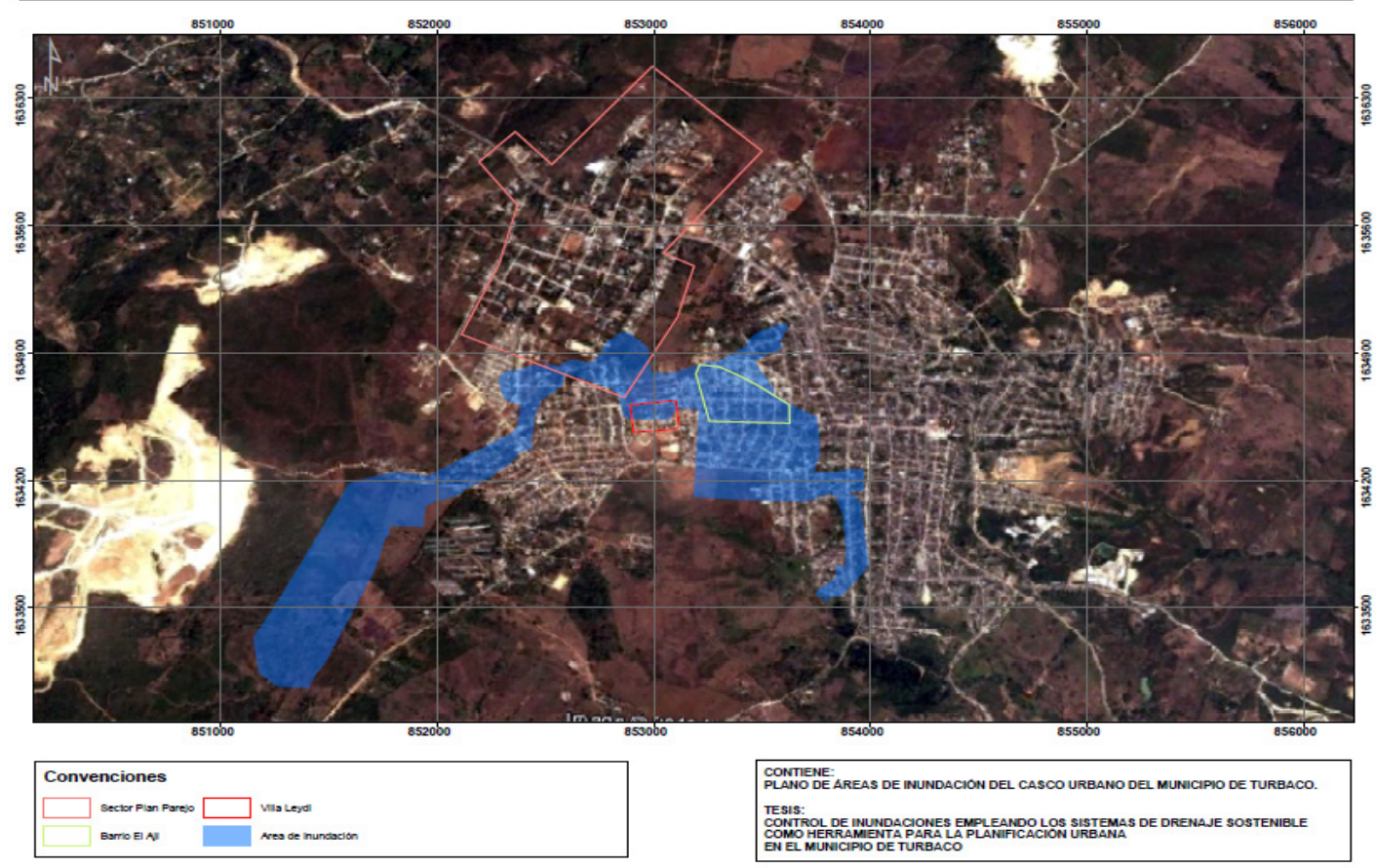

Figura 1. Área de inundación municipio de Turbaco - Bolívar.

Fuente: Proyecto Plan Prioritario de Drenaje Municipio de Turbaco, 2011

de las urbanizaciones no planificadas en el sector; lo anterior es evidente al observar las diferentes áreas urbanizadas en los barios el Ají, Villa Leydi y la Victoria. Otros factores no menos importantes son el taponamiento y contaminación con basuras de los canales naturales y la desforestación que realizan a las afueras del casco urbano, producto de la actividad minera (explotación de canteras).
Inundaciones ocurridas durante la época invernal del 2010 y 2011 (Figuras 2, 3 y 4) paralizaron y afectaron el municipio. De acuerdo al estudio realizado entre la Universidad de Cartagena y Cardique, el costo de reparación por vivienda fue de $\$ 7.461 .752$ (Cardique \& Universidad de Cartagena 2011).

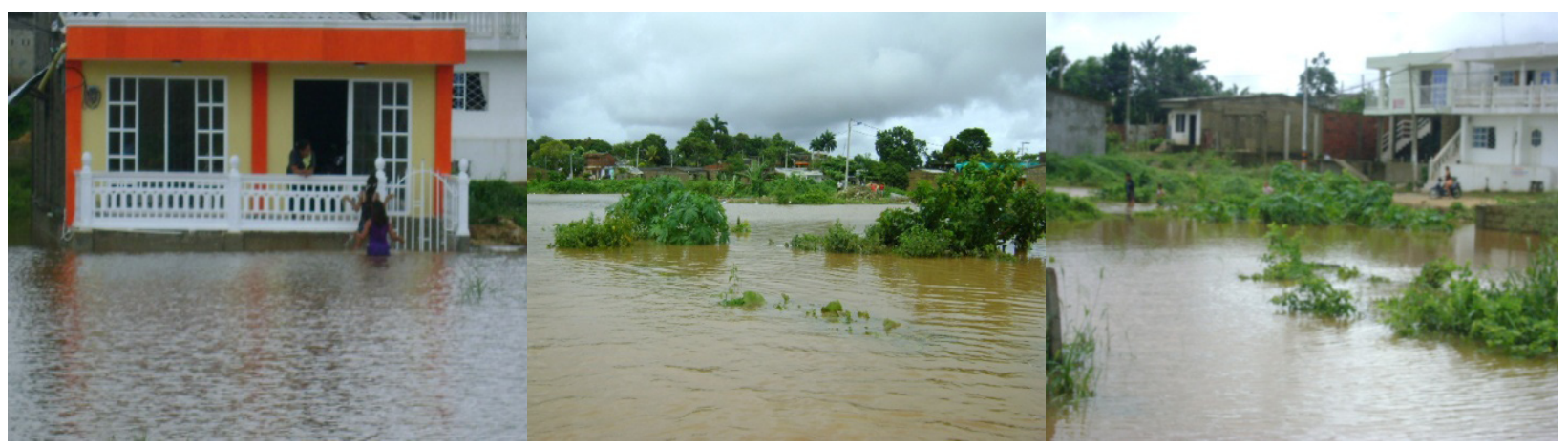

Figura 2. Inundación sector El Ají, año 2010.

Fuente: Proyecto Plan Prioritario de Drenaje del Municipio de Turbaco. 2011 


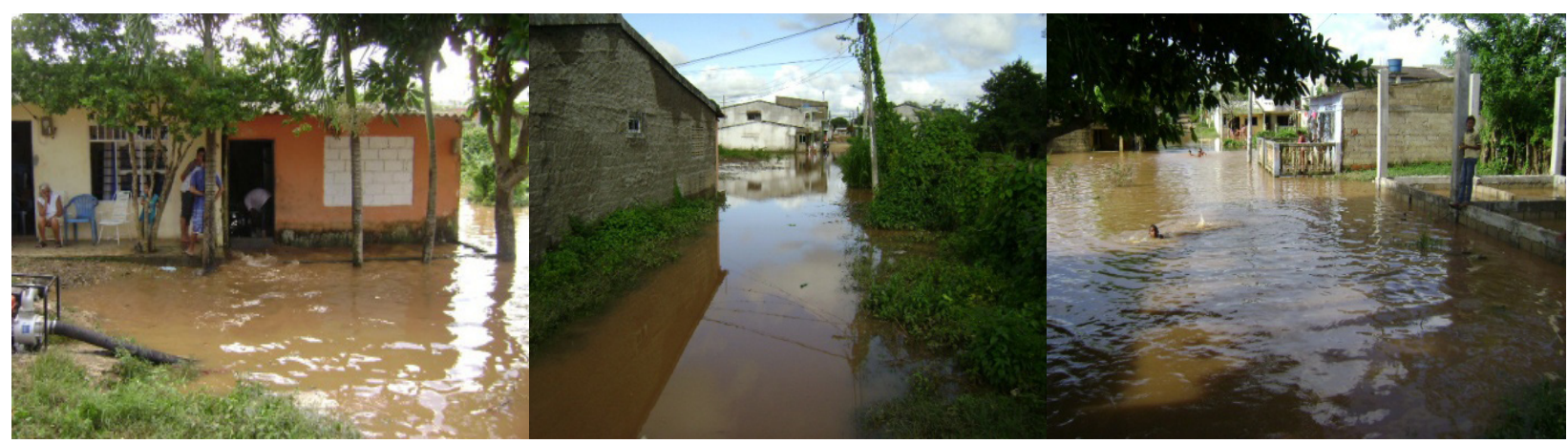

Figura 3. Inundación sector Villa Leydi, año 2010.

Fuente: Proyecto Plan Prioritario de Drenaje del Municipio de Turbaco, 2011

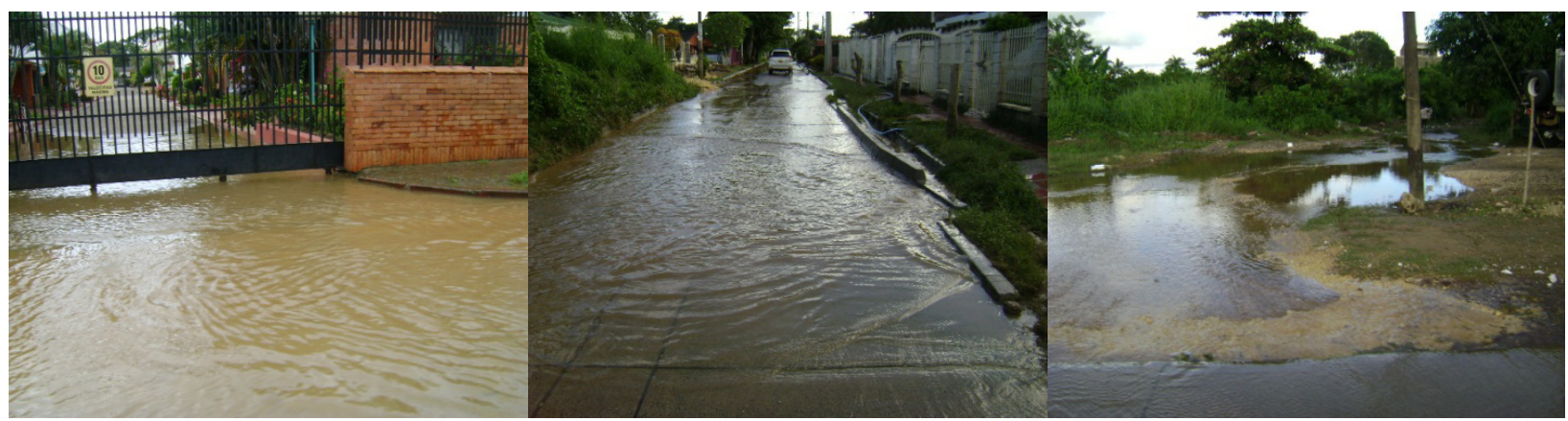

Figura 4. Inundación sector Plan Parejo, año 2010.

Fuente: Proyecto Plan Prioritario de Drenaje Municipio del Turbaco, 2011.

\section{CAMBIOS A NIVEL URBANO}

A nivel urbano los cambios son notables, estos son evidentes si observamos la evolución que ha tenido el perímetro del casco urbano del municipio (ver figura 5).

Durante los años 2012 a 2015 en el casco urbano del municipio se construyeron varios proyectos urbanísticos los cuales han influido en el cambio natural del suelo.

Entre las urbanizaciones construidas están Altos de Plan Parejo I y II, ubicadas al sur del sector de Plan Parejo, urbanización Las Heliconias ubicadas en la parte norte del sector de Plan Parejo y varias construcciones fueron llevadas a cabo en este periodo en los barrios Ají, Villa Leidy (figura 6).

\section{ANALISIS HIDROLÓGICO Y ESTIMACIÓN DE CAUDALES}

Para el cálculo de los modelos hidrológico e hidráulico se utilizó el modelo SWMM. El
Stormwater Management Model (Modelo de gestión de aguas pluviales) de la EPA (SWMM) es un modelo dinámico de simulación de precipitaciones. El programa permite simular tanto la cantidad como la calidad del agua evacuada, especialmente en alcantarillados urbanos. El módulo de escorrentía o hidrológico de SWMM funciona con una serie de cuencas en las cuales cae el agua de la lluvia y se genera la escorrentía (LIMUSA EPA 2005).

Para el caso de estudio, se utilizó como datos de entrada al modelo los registros de precipitación máxima de la estación Rafael Núñez por ser la estación pluviográfica más cercana a la zona de estudio con distribución de lluvia. Adicional a la información hidrológica, se contó con información topográfica del casco urbano (Cardique \& Universidad de Cartagena 2011), el cual permitió trazar el drenaje y las subcuencas del área de estudio. 


\section{EVOLUCIÓN HISTÓRICA DEL CASCO URBANO DEL MUNICIPIO DE TURBACO}
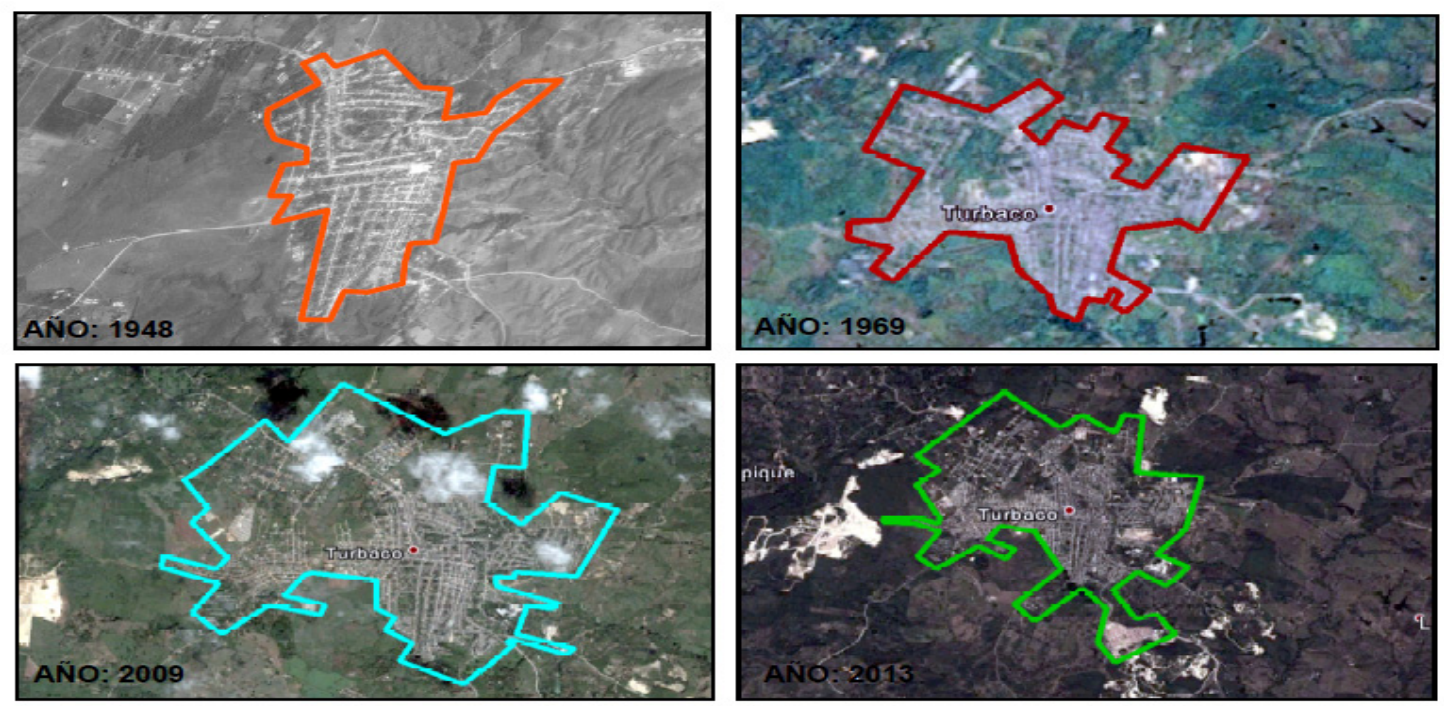

CONTIENE: IMAGENES DE LA EVOLUCIÓN HISTÓRICA DEL CASCO URBANO DEL MUNICIPIO DE TURBACO

TESIS:

CONTROL DE INUNDACIONES EMPLEANDO LOS SISTEMAS DE DRENAJE SOSTENIBLE COMO HERRAMIENTA PARA LA PLANIFICACIÓN URBANA EN EL
MUNICIPIO DE TURBACO

Figura 5. Evolución histórica del perímetro del municipio de Turbaco

Fuente: Google earth modificado por el autor. (Guerrero, 2014)

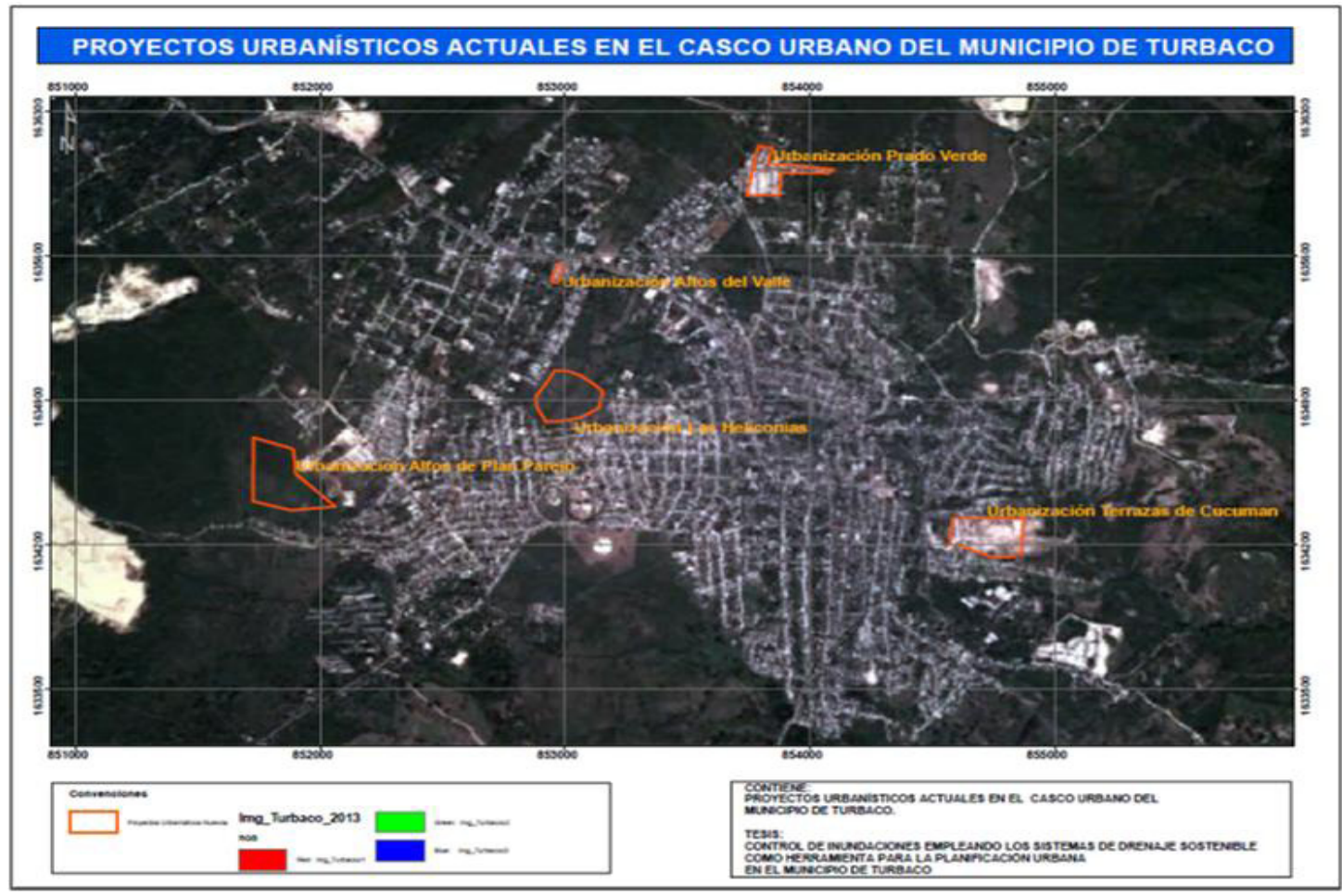

Figura 6. Proyectos urbanísticos desarrollados en el casco urbano de Turbaco Fuente: Google earth modificado por el autor. (Guerrero, 2014) 
Guerrero, M. - Embalses de retención y canales verdes: herramienta para el control de innundaciones.

Una vez se tienen los parámetros hidrológicos en el modelo, se introducen los parámetros hidráulicos; el software permite dibujar las estructuras que hacen parte del sistema de drenaje. Para el caso, se dibujaron los canales, los cuales fueron introducidos al software a través de nodos en el que el nodo inicial representa el inicio del canal y el nodo final su terminación. La longitud del canal estaba dada por la distancia entre los nodos y las cotas; entre los nodos se le asignaban cotas de acuerdo con la topografía. Posteriormente se asignaban los parámetros hidráulicos, lo cual incluyó la sección del canal y el tipo de material de revestimiento; para este caso de estudio se propuso una sección trapezoidal revestida en hierba.

Al sistema también se le dibujó la estructura de retención (embalse de retención) a la cual se le asignó el parámetro de relación de altura del embalse/área del espejo de agua. Para unir los canales con el embalse se crean unas transiciones, las cuales están representadas por el orificio y el vertedero del embalse

Para la modelación hidrológica se dividió el área de drenaje en subcuencas, con las cuales se pudieron estimar los caudales tributarios.

Durante el trazado y delimitación de subcuencas se obtuvieron 5 cuencas involucradas en la zona con posible riesgo de inundación (figura 7 y tabla 1 ).

Tabla 1. Áreas de subcuencas

\begin{tabular}{cc}
\hline Cuenca & Área (ha.) \\
\hline 1 & 115.2 \\
2 & 60.5 \\
3 & 67.8 \\
4 & 72.8 \\
5 & 90.3 \\
\hline
\end{tabular}

Fuente: Guerrero, 2014

Con los registros de precipitación de la estación aeropuerto Rafael Núñez se realizó el análisis de lluvias máximas. Para el estudio consistieron en la determinación de las lluvias máximas en 24 horas para diferentes períodos de retorno. La lluvia de diseño, fue escogida para un periodo retorno de 10 años y con una duración de 6 horas, teniendo en cuenta los tiempos de concentración de las cuencas ( Tabla 2).

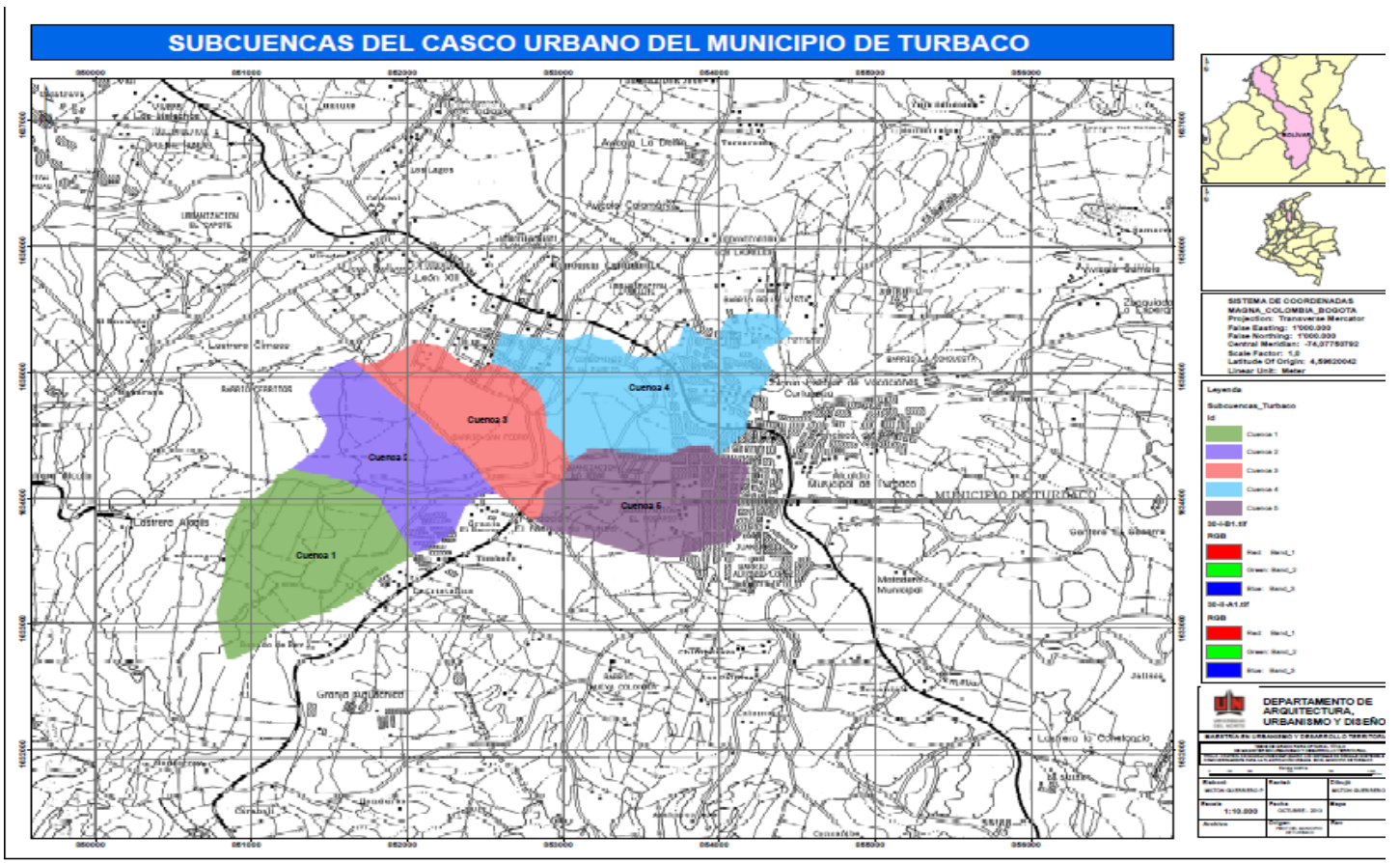

Figura 7. Subcuencas Casco urbano Municipio de Turbaco Bolívar.

Fuente: Guerrero, 2014 
Tabla 2. Lluvia para un periodo de retorno de 10 años

\begin{tabular}{cc}
\hline Tiempo $(\mathrm{HH}: \mathrm{MM})$ & Precipitación $(\mathrm{mm})$ \\
\hline $00: 00$ & 11.42 \\
$00: 15$ & 11.42 \\
$00: 30$ & 8.58 \\
$00: 45$ & 6.69 \\
$01: 00$ & 6.54 \\
$01: 15$ & 5.92 \\
$01: 30$ & 5.92 \\
$01: 45$ & 4.87 \\
$02: 00$ & 4.61 \\
$02: 15$ & 4.56 \\
$02: 30$ & 4.48 \\
$02: 45$ & 4.48 \\
$03: 00$ & 4.26 \\
$03: 15$ & 4.26 \\
$03: 30$ & 3.27 \\
$03: 45$ & 2.61 \\
$04: 00$ & 2.44 \\
$04: 15$ & 1.76 \\
$04: 30$ & 1.76 \\
$04: 45$ & 1.88 \\
$05: 00$ & 1.92 \\
$05: 15$ & 1.63 \\
$05: 30$ & 1.19 \\
$05: 45$ & 1.19 \\
$06: 00$ &
\end{tabular}

Fuente: Guerrero, 2014

\section{RESULTADOS}

Como resultado de la modelación se obtuvo el hidrograma total (Figura 8), el cual muestra los caudales obtenidos para las cuencas aferentes al canal principal que será proyectado.

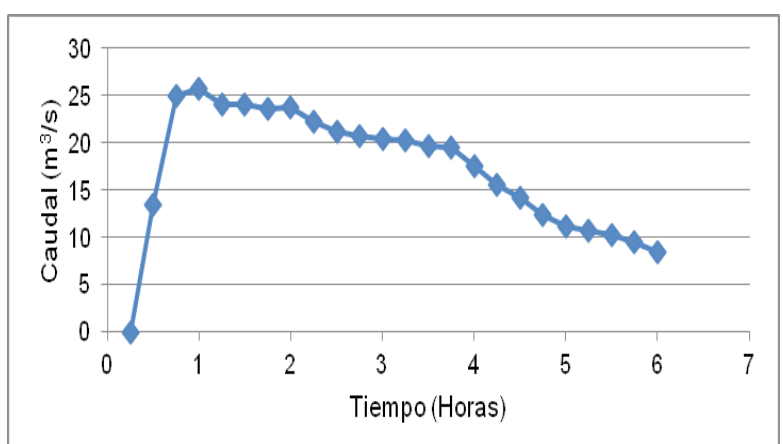

Figura 8. Hidrograma total para un periodo de retorno de 10 años

Fuente: Guerrero, 2014
La figura 9 muestra los hidrogramas para cada cuenca para un periodo de retorno de 10 años.

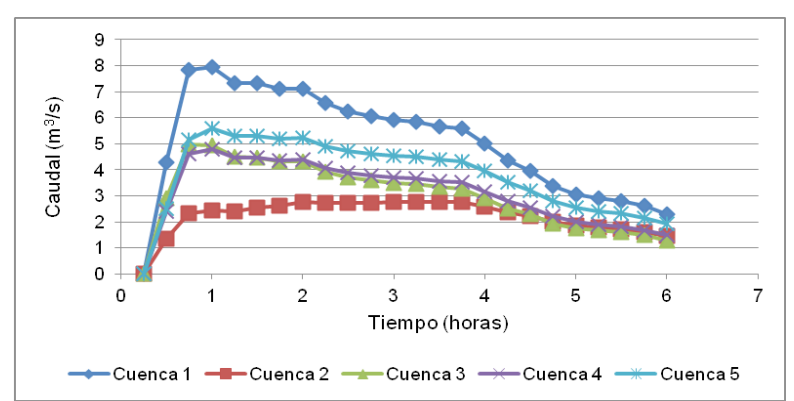

Figura 9. Hidrogramas para un periodo de retorno de 10 años.

Fuente: Guerrero, 2014

Los resultados anteriores muestran los hidrogramas de las cuencas sin realizar ninguna intervención.

Con el objetivo de dar solución al problema de inundación se proyectó un embalse de retención y canales trapezoidales revestidos en hierba. Con el embalse se amortiguan las crecientes de inundación y se disminuyen las secciones de los canales con lo que se evita de esta forma la reubicación de viviendas. Esta estructura de retención estará integrada al espacio urbano del municipio. El embalse, retendrá el caudal que aporta la cuenca 1 y lo descargará gradualmente produciendo una disminución en el pico del hidrograma total (Figura 10).

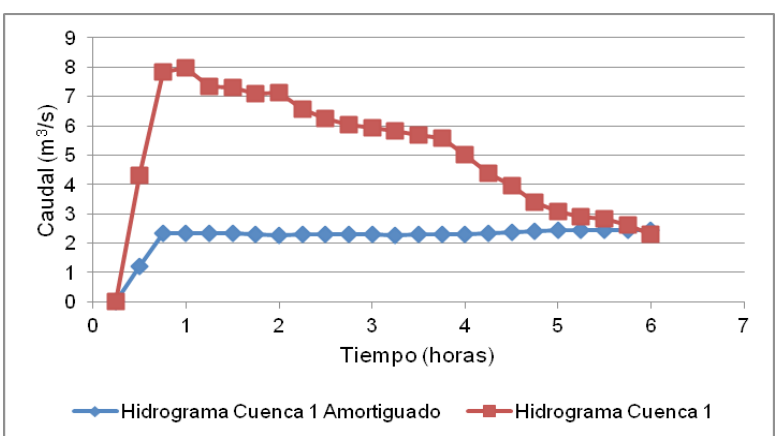

Figura 10. Hidrograma de amortiguamiento de la cuenca 1 a través del embalse.

Fuente: Guerrero, 2014

Es importante la ubicación y dimensionamiento del embalse como estructura de amortiguación (Figuras 11, 12 y 13), en este caso se consideró la localización de la cuenca 1(Figura 7), y el 


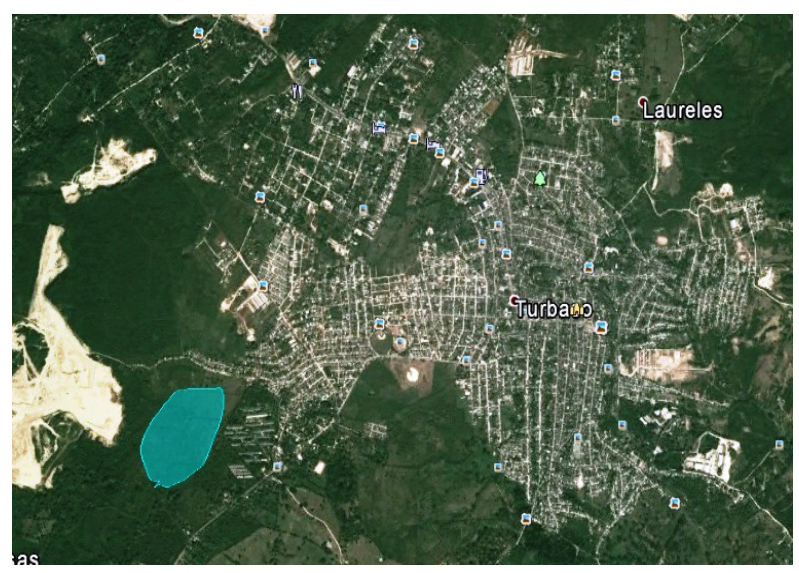

Figura 11. Ubicación de estructura de almacenamiento. Fuente: gogle earth. Modificado por el autor. Guerrero, 2014

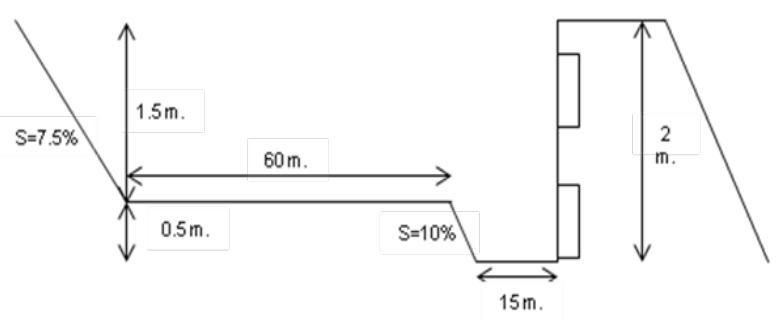

Figura 12. Perfil embalse de retención Fuente: Guerrero, 2014

Considerando las áreas aledañas al embalse como futuras intervenciones urbanísticas, se desarrolló el concepto del área embalse como un área que permita la comunicación, el esparcimiento y la recreación para sus habitantes. Por esta razón se concibió como una zona donde se puede dar la práctica de deportes náuticos y su área perimetral fue concebida como áreas propicias para la comunicación y la integración de la comunidad (Figuras 14, 15 y16).

Complementario al embalse se proyectan dos canales en hierba de sección trapezoidal. Un canal principal y otro secundario. El primero se proyecta a lo largo de la corriente principal del drenaje natural. Este canal recoge agua del sector y lo descarga en el arroyo Mameyal, y este a su vez en el arroyo Grande. El canal secundario se proyectó para recoger toda la escorrentía pluvial del sector del Ají, descargando en el canal principal. El trazado de este canal se realizó a través de la corriente del drenaje natural. Con el fin de conservar el concepto urbanístico se adoptó una sección área para proponer la ubicación del embalse. El embalse se proyectó ubicarlo en un área contigua al barrio La Victoria, se determinó esta área dadas las características topográficas del terreno, propicio para el desarrollo de este tipo de sistema de drenaje. El embalse tiene como función retener la escorrentía pluvial proveniente de la zona sur oeste del municipio y retener la carga de sedimentos. El embalse tiene un área de una (1ha.) hectárea y una profundidad máxima de $2 \mathrm{~m}$. (Figuras 12 y 13 ).

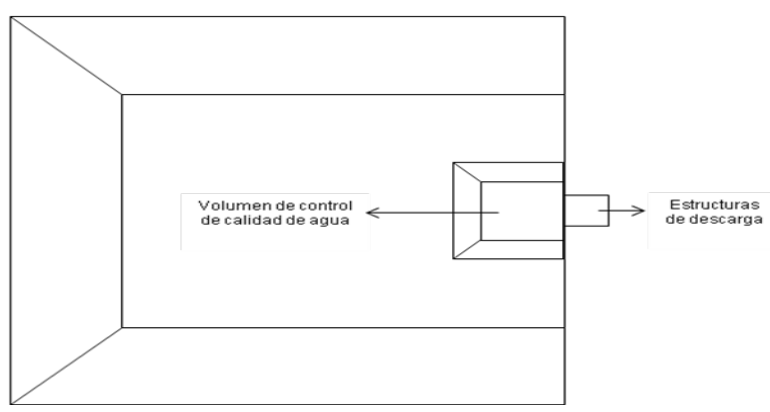

Figura 13. Vista en planta del embalse.

Fuente: Guerrero, 2014

trapezoidal y un coeficiente de rugosidad de 0.03 que corresponde a un revestimiento vegetal del canal. En el sector del Ají se diseñó un canal trapezoidal revestido con el fin de recoger la escorrentía de la zona y darle mayor capacidad de evacuación al sistema (Figura 17).

Considerando que los canales también hacen parte de la integración del paisaje urbano, y que estos recorren las calles del casco urbano del municipio, se proponen calles con superficies permeables laterales al canal (Figuras 18, 19 y 20). Esta superficie lateral permitirá el tránsito de vehículos y peatones, como también la percolación de las aguas lluvias a superficies inferiores de la estructura.

\section{CONCLUSIONES}

La capacidad de retención del embalse es del $25 \%$ del caudal total. Este porcentaje de retención permitirá controlar las inundaciones del sector de los barrios La Victoria, El Ají, Villa Leidy y Plan Parejo.

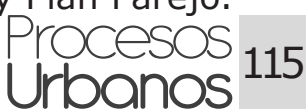




\section{Procesos Urbanos N³ Enero - Diciembre; 2016}

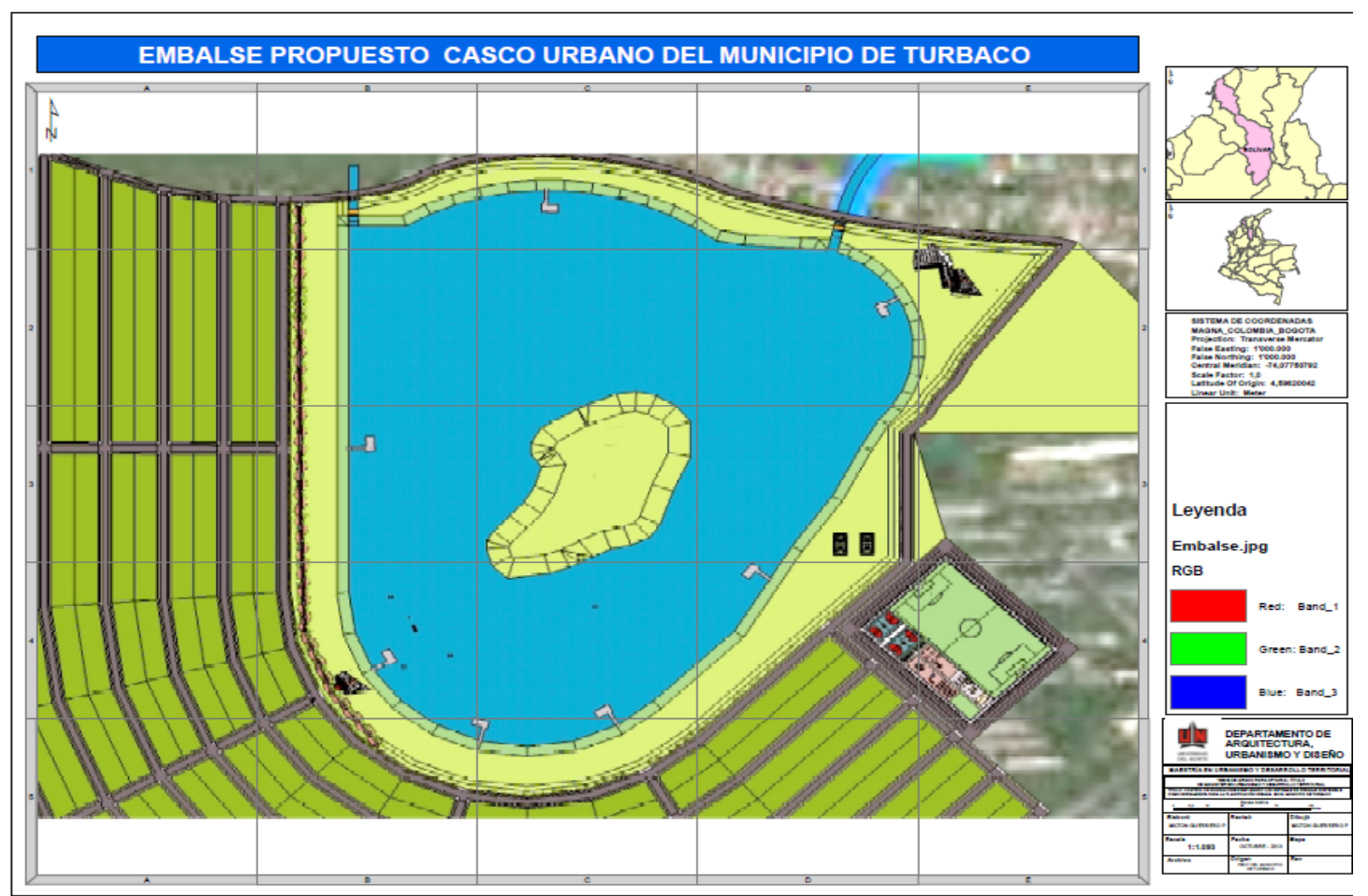

Figura 14. Embalse de retención.

Fuente: Guerrero, 2014

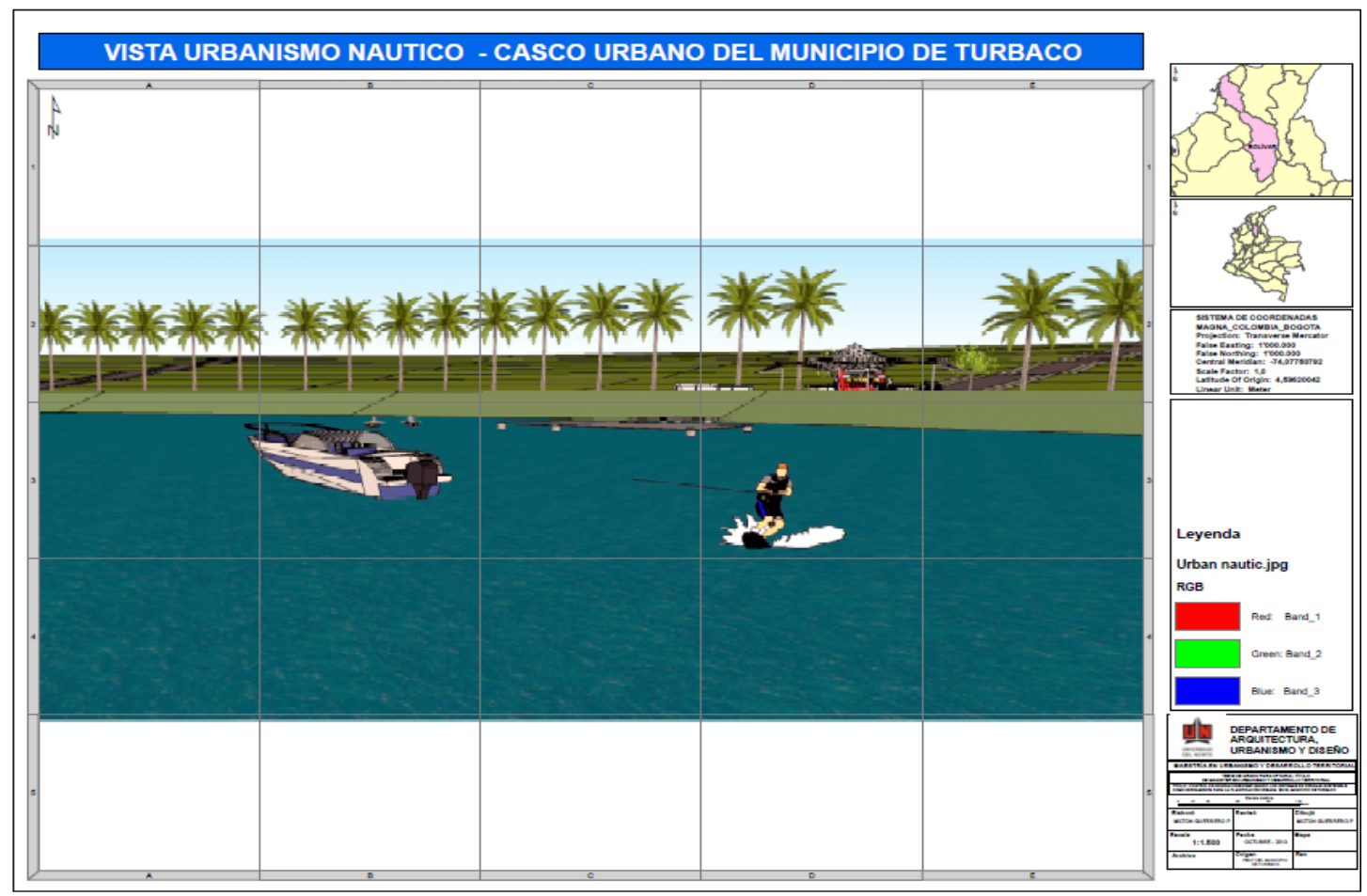

Figura 15. Embalse para prácticas de deportes náuticos.

Fuente: Guerrero, 2014 
Guerrero, M. - Embalses de retención y canales verdes: herramienta para el control de innundaciones.

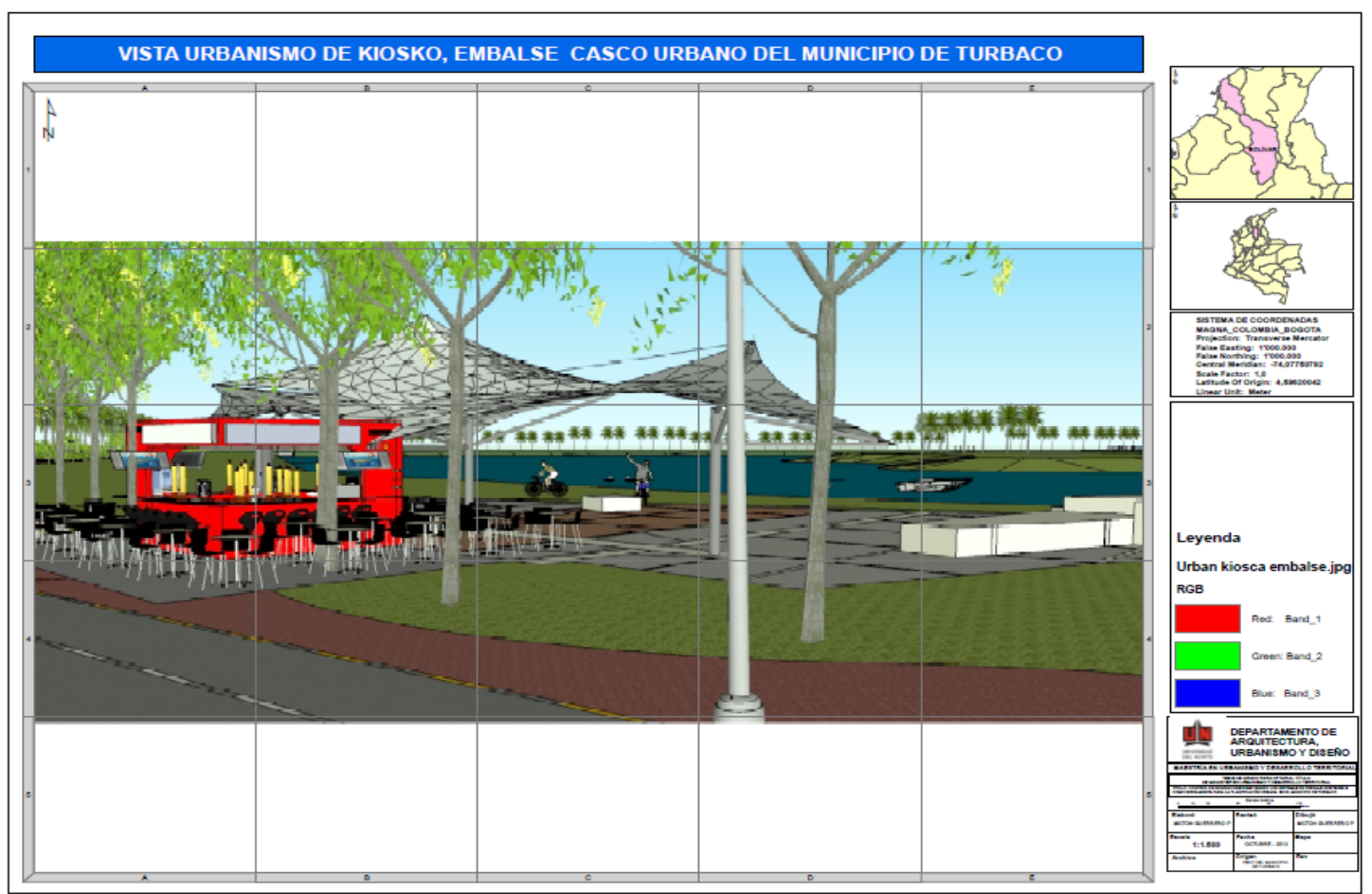

Figura 16. Áreas sociales alrededor del embalse. Fuente: Guerrero, 2014

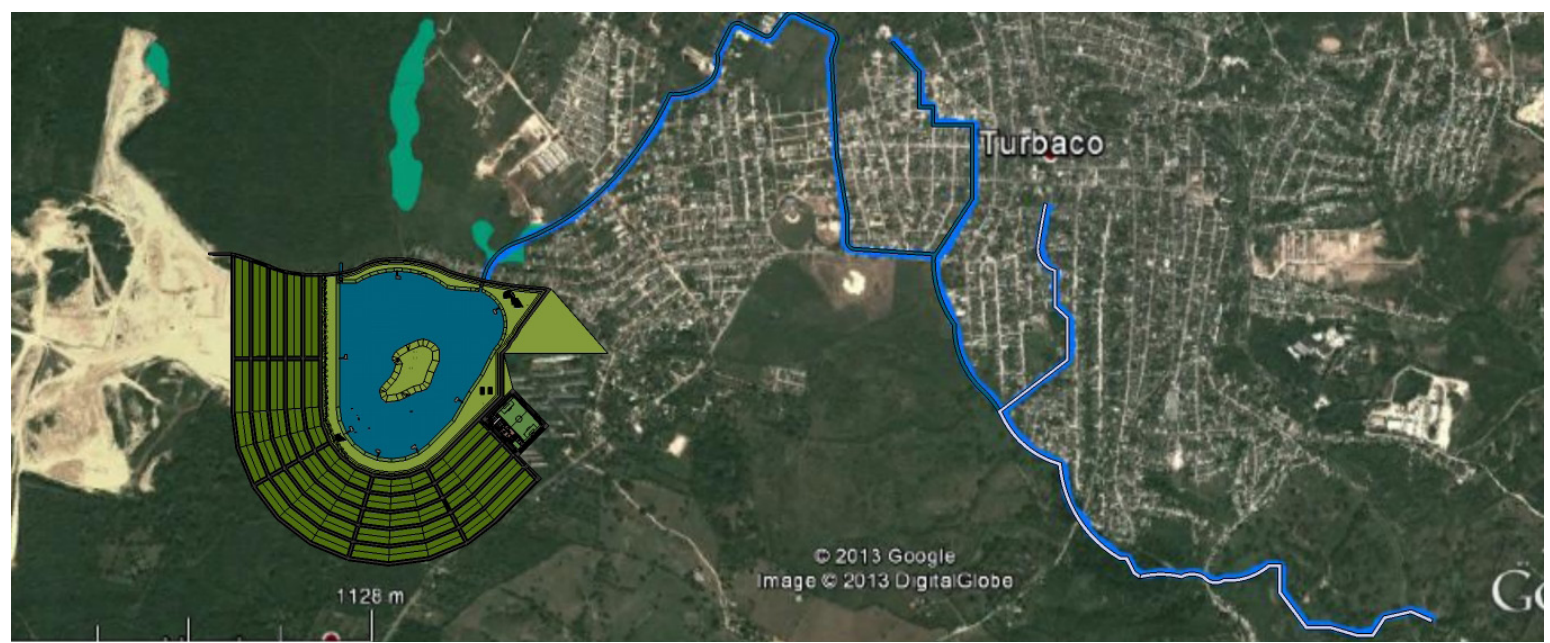

Figura 17. Localización de embalse y canales. Fuente: google earth. Modificado por el autor. Guerrero, 2014 
Procesos Urbanos № 3 Enero - Diciembre; 2016

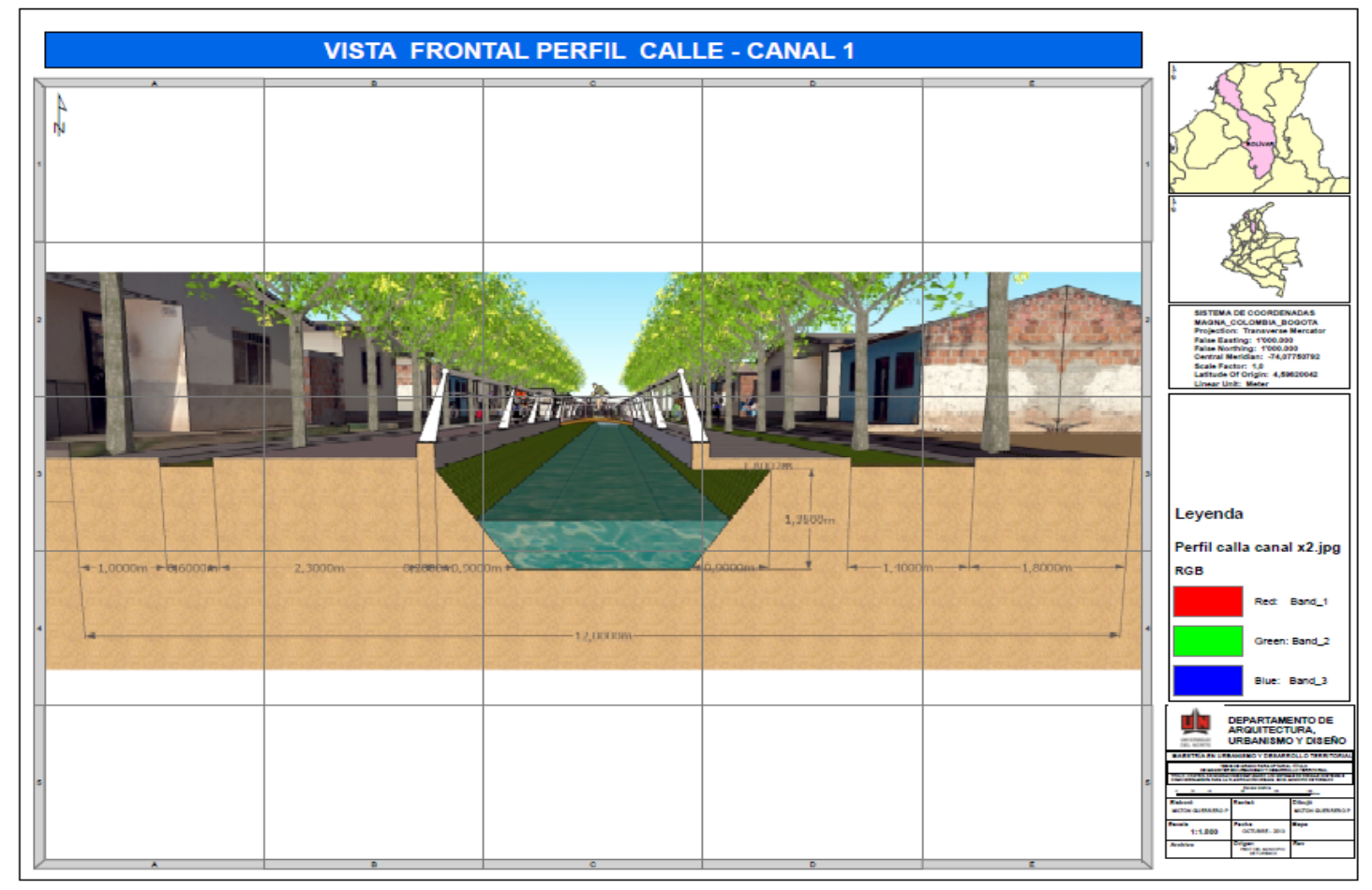

Figura 18. Canal trapezoidal en hierba Fuente: Guerrero, 2014

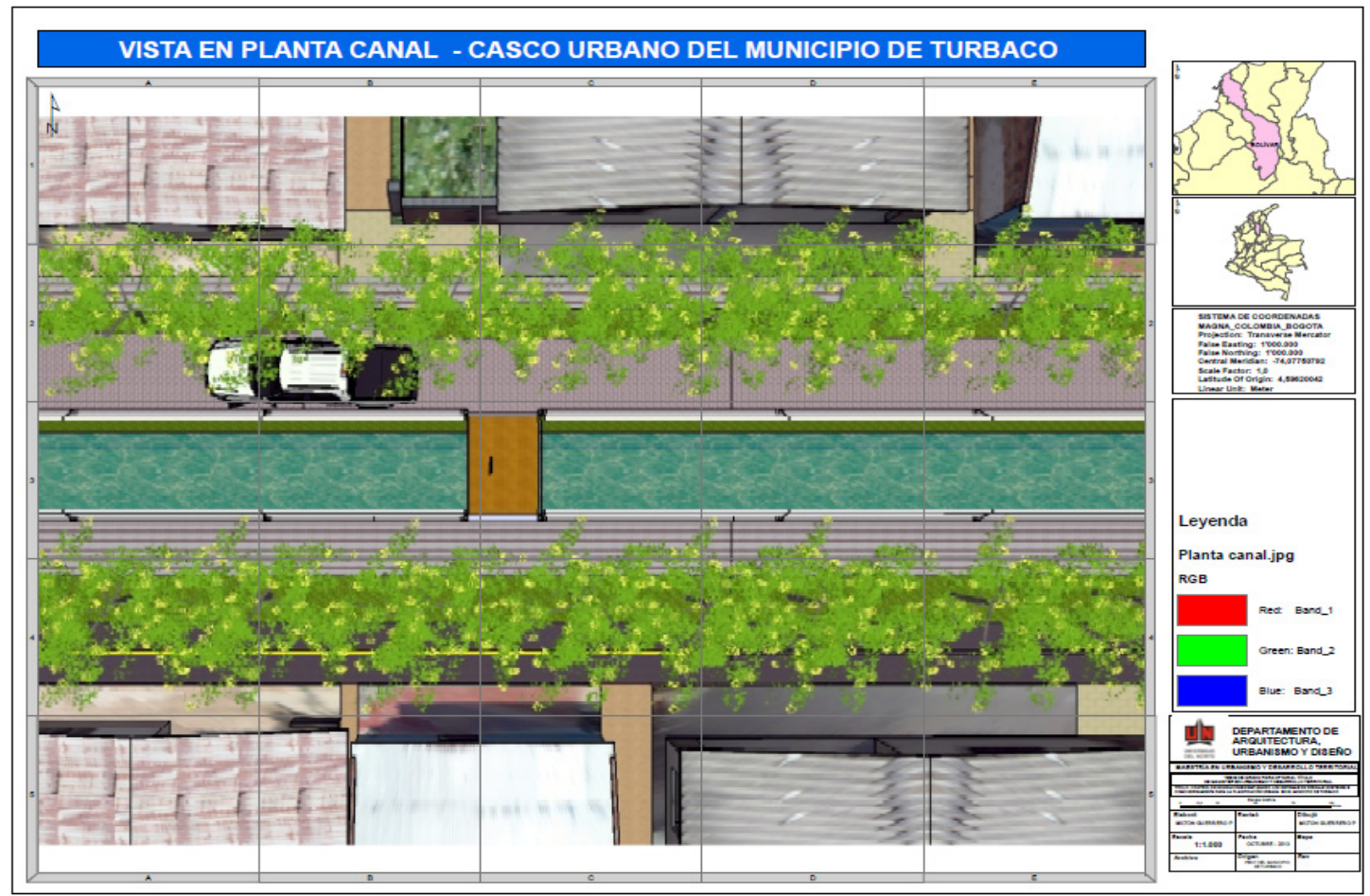

Figura 19. Vista en planta del canal Fuente: Guerrero, 2014 


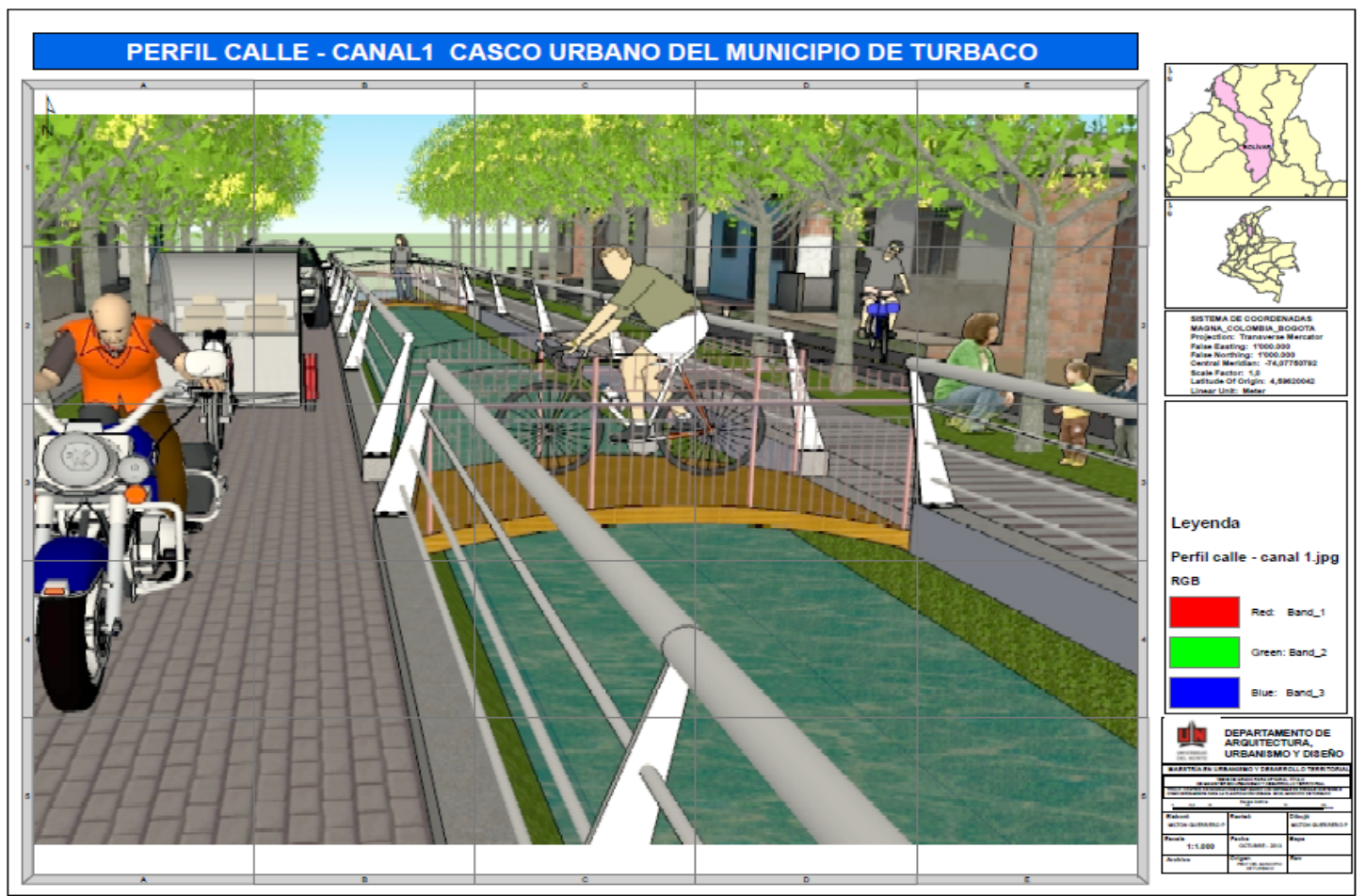

Figura 20. Propuesta perfil de calles municipio de Turbaco Fuente: Guerrero, 2014

Los factores que más influyen en las inundaciones son: la falta de un alcantarillado pluvial, la obstrucción de canales de evacuación, la impermeabilización de los suelos, producto de los procesos urbanísticos que se han llevado a cabo en el municipio y la explotación minera.

La propuesta del embalse y canales revestidos en hierba hacen parte de la propuesta del sistema urbano de drenaje sostenible, SUDS del municipio y se presentan como un elemento integrador municipio-naturaleza que debería ser considerada como herramienta para la planificación urbana.

Los nuevos proyectos urbanos que se desarrollen en el municipio deberían considerar la implementación de los SUDS (estanques de retención y cunetas verdes, zanjas de infiltración, superficies permeables) como elementos para el manejo de la escorrentía superficial

\section{REFERENCIAS}

ALCALDIA MUNICIPAL DE TURBACO, BOLIVAR, (2002) Plan Básico de Ordenamiento Territorial, agosto de 2002.

AYUNTAMIENTO DE MADRID. Ordenanza de Gestión y Uso Eficiente del Agua en la ciudad de Madrid. Madrid. 1-108.

CORPORACIÓN AUTÓNOMA DEL CANAL DEL DIQUE (CARDIQUE) - UNIVERISDAD DE CARTAGENA, (2011) Plan Prioritario de Drenaje 2011.

Guerrero Pájaro, M. (2014) Tesis de maestría. Control de inundaciones empleando los sistemas de drenaje sostenible como herramienta para la planificación urbana. Universidad del Norte 2014.

LIMUSA.EPA. (2005). Manual del usuario SWMM 5.0. 